\title{
AVALIAÇÃO MICROBIOLÓGICA DE SORVETES COMERCIALIZADOS NA CIDADE DE POUSO ALEGRE - MG.
}

\section{MICROBIOLOGICAL EVALUATION OF ICE CREAM SOLD IN THE CITY OF POUSO ALEGRE - MG.}

\author{
Mayra de Oliveira Silva ${ }^{1}$ \\ Universidade do Vale do Sapucaí, UNIVÁS. Pouso Alegre, MG, Brasil. \\ https://orcid.org/0000-0003-3758-5607 \\ mayraoli_09@hotmail.com \\ Luiz Francisley de Paiva ${ }^{2}$ \\ Universidade do Vale do Sapucaí, UNIVÁS. Pouso Alegre, MG, Brasil. \\ https://orcid.org/0000-0001-6497-7468 \\ francisleybiologo@yahoo.com.br \\ Carlos Américo Veiga Damasceno ${ }^{2}$ \\ Universidade de Itaúna. Itaúna, MG, Brasil. \\ https://orcid.org/ 0000-0001-9635-6447 \\ cavdamasceno@uol.com.br
}

\footnotetext{
${ }^{1}$ Conceptualização, metodologia, curadoria de dados, primeira redação, revisão e aprovação da versão final do trabalho.

2 Administração do projeto, análise formal, conceptualização, metodologia, curadoria de dados, revisão e aprovação da versão final do trabalho.
}

\section{RESUMO}

O presente estudo teve como objetivo avaliar a qualidade microbiológica de sorvetes comercializados na cidade de Pouso Alegre - MG. Foram adquiridas 2 amostras de sorvete de 18 sorveterias em um intervalo de 30 dias totalizando 36 amostras. As amostras de sorvetes foram submetidas às análises para enumeração de coliformes totais, coliformes termotolerantes, Estafilococos coagulase positiva e Salmonella spp. As análises foram realizadas segundo os protocolos da American Public Health Association descrita no Compendium of methods for the microbiological examination of foods. Neste estudo não foram encontradas espécies de Salmonella spp. entretanto, houve amostras contaminadas por E. coli e Estafilococos coagulase positiva.13,89\% das amostras analisadas estavam fora dos padrões estabelecidos. Verificou-se ainda a presença de coliformes totais em $77,78 \%$ e de Staphylococcus sp. em $36,11 \%$ e, embora a legislação vigente não mencione limites de contaminação para estes microrganismos, pode-se dizer que a matéria-prima estava contaminada, que as condições higiênicas, no processamento e armazenamento estavam inadequados para este produto. As amostras analisadas obtidas de sorveterias da região central foram as mais contaminadas, onde foi encontrada a presença de E. coli e Estafilococos coagulase positiva. A presença desses microrganismos sugere falhas em algum momento do processamento, armazenamento ou da venda destes produtos tornando-os inadequados, 
podendo não só causar uma deterioração mais rápida do sorvete, mas também colocando em risco a saúde do consumidor.

Palavras-chave: Escherichia coli. Segurança Alimentar Sanitária. Staphylococcus.

\section{ABSTRACT}

This study aimed to evaluate the microbiological quality of ice cream sold in the city of Pouso Alegre - MG. Two ice cream samples were acquired from 18 ice cream parlors in a 30-day interval, totaling 36 samples. The ice cream samples were subjected to analysis for enumeration of total coliforms, thermotolerant coliforms, coagulase positive Staphylococci and Salmonella spp. The analyzes were performed according to the protocols of the American Public Health Association described in the Compendium of Methods for the Microbiological Examination of Foods. In this study no species of Salmonella spp. were found, however, there were samples contaminated by $E$. coli and coagulase positive Staphylococci. $13.89 \%$ of the analyzed samples were outside the established standards. It was also verified the presence of total coliforms in $77.78 \%$ and Staphylococcus sp. in $36.11 \%$ and, although the current legislation does not mention contamination limits for these microorganisms, it can be said that the raw material was contaminated, that the hygiene, processing and storage conditions were inadequate for this product. The analyzed samples obtained from ice cream parlors in the central region were the most contaminated, where the presence of $\mathrm{E}$. coli and coagulase positive Staphylococci was found. The presence of these microorganisms suggests failures at some point in the processing, storage or sale of these products, making them unsuitable and not only causing a faster deterioration of the ice cream, but also putting the consumer's health at risk.
Keywords: Escherichia coli. Food Safety. Staphylococcus.

INTRODUÇÃO

O sorvete é uma sobremesa que tem sido muito consumida no Brasil e teve um aumento considerável na produção nacional a partir de 2016. Números da Associação Brasileira da Indústria de Alimentos (ABIA) mostram que em 2016 foram produzidos 1.065 milhões de litros de sorvete. Já em 2017 o número cresceu para 1.068 e em 2018 fechou o ano em 1.103 milhões de litros. O consumo per capita também cresceu de 5,2 litros em 2016 para 5,3 litros em 2018 (RHA, 2019).

Os principais consumidores de sorvetes são as crianças, incluindo aquelas com idade em que o sistema imune ainda é imaturo, e que por isso se deve ter o máximo de cuidado durante a escolha da matéria-prima que será utilizada e das etapas de processamento. O leite é o principal ingrediente do sorvete e contém vários nutrientes benéficos à saúde, como as vitaminas $A, B 1, B 2, B 6$, $\mathrm{C}, \mathrm{D}, \mathrm{K}, \mathrm{O}$ cálcio, fósforo, as proteínas, açúcares, gordura vegetal e/ou animal, que são essenciais numa proporção equilibrada (OLIVEIRA et al., 2012).

A Agência Nacional de Vigilância Sanitária (ANVISA) define gelados comestíveis, como produtos alimentícios obtidos a partir de uma emulsão de gorduras e proteínas, com ou sem adição de outros ingredientes e substâncias, ou de uma mistura de água, açúcares e 
outros ingredientes que tenham sido congelados, em condições que garantam a conservação do produto congelado durante o armazenamento, transporte, comercialização e entrega do produto (BRASIL, 2003).

O controle microbiológico é de fundamental importância para não ocorrer uma disseminação de microrganismos causadores de toxinfecções, porque depois do preparo final, não há como submeter o sorvete em um processo de cocção ou esterilização, como acontece em outros alimentos (FALCAO et al., 1983). Os microrganismos encontrados no produto podem estar relacionados com os ingredientes utilizados na fabricação, ou no manuseio inadequado nas operações de processamento, embalagem e condições de armazenamento (OLIVEIRA et al., 2012).

Já foi constatado que, mesmo que o sorvete seja mantido em baixa temperatura, não é o suficiente para inibir o crescimento bacteriano, pois todos os alimentos gelados e comestíveis feitos à base de laticínios e ovos devem ser pasteurizados (DIOGO et al., 2002).

Para evitar a contaminação do sorvete é necessário não somente escolher matéria prima de boa qualidade, mas também utilizar a pasteurização ou outro tratamento térmico para reduzir 0 número de microrganismos patogênicos. Também é de total importância a prática de um monitoramento dos chamados pontos críticos de controle, para não aumentar a chance de outros contaminantes (DIOGO et al., 2002).

Considerando a necessidade de constante aperfeiçoamento das ações de controle sanitário na área de alimentos, visando à proteção à saúde da população, a ANVISA determina a regulamentação dos padrões microbiológicos para alimentos onde a tolerância é máxima e os padrões são mínimos para os diferentes grupos de produtos alimentícios (BRASIL, 2001). Desta maneira, o presente estudo teve como objetivo avaliar a qualidade microbiológica de sorvetes comercializados na cidade de Pouso Alegre - MG.

\section{METODOLOGIA}

\section{Amostras}

Foram analisadas em duplicata 36 amostras de sorvetes no período de março a agosto de 2014, sendo 18 amostras no primeiro dia e 18 amostras após 30 dias. O sabor escolhido foi o de chocolate. As sorveterias escolhidas para pesquisa estão localizadas: 7 na região central da cidade, 4 nas regiões periféricas e 7 dentro de um grande centro comercial na cidade de Pouso Alegre MG. Cada sorveteria recebeu uma letra alfabética para manter a sua identidade e integridade. 
No momento da aquisição, aproximadamente $150 \mathrm{~g}$ do sorvete foi colocado diretamente em frascos estéreis pelo vendedor. Todas as amostras foram mantidas e transportadas refrigeradas em caixas isotérmicas, para o Laboratório de Pesquisas Básicas da Universidade do Vale do Sapucaí - Univás. A realização dos testes não excedeu 2 horas após a aquisição.

\section{Análises microbiológicas}

As amostras de sorvetes foram submetidas às análises para enumeração de coliformes totais, coliformes termotolerantes, Estafilococos coagulase positiva e Salmonella spp. As análises foram realizadas segundo os protocolos da American Public Health Association descrita no Compendium of methods for the microbiological examination of foods (APHA, 2015).

Inicialmente foram pesadas $25 \mathrm{~g}$ dos sorvetes e transferidas para um erlenmeyer contendo $225 \mathrm{~mL}$ de água peptonada $0,1 \%$. As amostras foram diluídas homogeneizando o frasco durante 5 minutos. A partir desta solução, realizouse diluições seriadas decimais de 1:10, 1:100 e 1:1000 (v/v).

Para a enumeração de coliformes totais e termotolerantes utilizou-se a técnica do Número Mais Provável (NMP). A análise presuntiva de coliformes foi realizada em Caldo Lauril Sulfato de Sódio
(LST), com incubação por 48 horas a $35^{\circ} \mathrm{C}$. A enumeração de coliformes totais foi efetuada em Caldo Lactosado Bile Verde Brilhante, com incubação a $35^{\circ} \mathrm{C}$ por 24 a 48 horas. A enumeração de coliformes termotolerantes foi realizada em Caldo Escherichia coli (EC) e em Caldo Triptona com incubação a $45,5^{\circ} \mathrm{C}$ por 24 horas em banho-maria.

Os tubos considerados como positivo apresentaram presença de gás nos caldos e de indol no Caldo Triptona a partir do aparecimento de coloração vermelho-escura após adição de reativo de Kovacs.

Para a verificação da presença de Salmonella spp. nas amostras inicialmente, realizou-se o préenriquecimento em água peptonada 0,1\% por 24 horas e em seguida foi transferido $1 \mathrm{~mL}$ para o Caldo Selenito Cistina e Caldo Tetrationato, ambos meios de enriquecimento seletivo. Os tubos foram incubados a $35^{\circ} \mathrm{C}$ por 24 horas.

Após esse período, uma alçada dos tubos com crescimento positivo foi inoculada em placas de Ágar Hectoen, Ágar Bismuto Sulfito e Ágar Xilose Lisina Desoxiciolato. As placas foram incubadas invertidas a $35^{\circ} \mathrm{C}$ por 24 horas. Após o período de incubação, foi verificado o desenvolvimento de colônias típicas e atípicas de Salmonella spp.

Colônias típicas e atípicas de $E$. coli e Salmonella spp. foram isoladas e 
purificadas em Agar MacConkey. Após esse processo, os microrganismos foram identificados por provas bioquímicas utilizando o Enterokit B - Probac do Brasil. O kit contém 3 meios e oferece 8 testes sendo eles a fermentação e produção de gás em glicose, produção de $\mathrm{H}_{2} \mathrm{~S}$, hidrólise da uréia, desaminação do triptofano, motilidade, descarboxilação da lisina, indol e utilização de citrato como única fonte de carbono. A incubação foi realizada a $35 \pm 2^{\circ} \mathrm{C}$ e a leitura após 24 horas. Os 8 testes somados ao da fermentação da lactose na placa de isolamento, permitem identificar grande maioria das enterobactérias.

Para as análises de Estafilococos coagulase positiva, foram inoculados 100 $\mu \mathrm{L}$ de cada diluição em placas contendo o meio Agar Baird Parker suplementado com emulsão gema de ovo e telurito de potássio $1 \%$. As placas foram identificadas e incubadas a 24-48 horas a $35^{\circ} \mathrm{C} \pm 2^{\circ} \mathrm{C}$ em triplicatas. Após esse período, as colônias típicas e também atípicas quando presentes, foram contadas e após, foram determinados 0 número de UFC/g.

Para a verificação de Estafilococos coagulase positiva, foram selecionadas cinco colônias de cada placa e transferidos para tubos contendo Caldo Infusão Cérebro Coração incubados a $35^{\circ} \mathrm{C} \pm 2^{\circ} \mathrm{C}$ por $18-24$ horas. Após esse período $0,2 \mathrm{~mL}$ da cultura foram transferidos para tubos contendo $0,5 \mathrm{~mL}$ de plasma de coelho com EDTA e incubados por 6 horas a $35^{\circ} \mathrm{C} \pm 2^{\circ} \mathrm{C}$ com verificação periódica. Foi avaliada a formação de coágulos quando presentes e atribuídos uma nota de nível que varia de $1+$ à 4+, sendo confirmativo para Estafilococos coagulase positiva os níveis $3+$ e $4+$.

\section{RESULTADOS E DISCUSSÃO}

Neste estudo, das 36 amostras analisadas, $\quad 77,78 \% \quad$ estavam contaminadas com coliformes totais e $72,23 \%$ com espécies do gênero Staphylococcus sp. Embora a resolução vigente, não estabelece padrões microbiológicos para estes microrganismos, é importante ressaltar que o aparecimento deste sugere falha durante a manipulação do alimento, portanto, pode ocorrer contaminação por outros patógenos. Neste estudo não foram encontradas espécies de Salmonella sp. estando em acordo com a resolução, entretanto, houve amostras contaminadas por E. coli e Estafilococos coagulase positiva.

Das amostras analisadas no primeiro dia pertencentes às sorveterias da região central da cidade, todas estavam contaminadas por coliformes totais e por estafilococos. Em três destas amostras a quantidade de microrganismos 
encontrados

ultrapassam os limites

uma estava contaminada com estabelecidos para coliformes Estafilococos coagulase positiva com termotolerantes e também para valores acima do permitido. Nas Estafilococos coagulase positiva como demostrado na tabela 1 . Já nas sorveterias do centro comercial apenas sorveterias da região periférica da cidade, não foram observados microrganismos patogênicos.

Tabela 1 - Qualidade microbiológica de sorvetes comercializados em sorveterias analisados no primeiro dia.

\begin{tabular}{|c|c|c|c|c|c|c|c|}
\hline \multirow{2}{*}{ Região } & \multirow{2}{*}{ Sorveteria } & \multicolumn{3}{|c|}{ Coliformes (NMP/g) } & \multirow{2}{*}{ Salmonella sp. } & \multicolumn{2}{|c|}{ Estafilococos (UFC/g) } \\
\hline & & Totais & Termo. & E. coli & & $\mathrm{CN}$ & $\mathbf{C P}$ \\
\hline \multirow{7}{*}{$\begin{array}{l}\text { Região } \\
\text { Central }\end{array}$} & A & 23 & $<3$ & - & Ausente & 400 & - \\
\hline & B & $\geq 1100$ & 240 & + & Ausente & 45100 & $451 \times 10^{2}$ \\
\hline & C & $\geq 1100$ & 240 & + & Ausente & 7533 & - \\
\hline & D & 93 & 9 & - & Ausente & 550 & - \\
\hline & $\mathbf{E}$ & 240 & $<3$ & - & Ausente & 300 & - \\
\hline & $\mathbf{F}$ & 23 & $<3$ & - & Ausente & 22900 & $229 \times 10^{2}$ \\
\hline & $\mathbf{G}$ & $\geq 1100$ & $<3$ & - & Ausente & 2450 & - \\
\hline \multirow{7}{*}{$\begin{array}{c}\text { Centro } \\
\text { Comercial }\end{array}$} & $\mathbf{H}$ & 240 & $<3$ & - & Ausente & 10000 & - \\
\hline & $\mathbf{I}$ & $<3$ & $<3$ & - & Ausente & - & - \\
\hline & $\mathbf{J}$ & 23 & $<3$ & - & Ausente & 72000 & $720 \times 10^{2}$ \\
\hline & $\mathbf{L}$ & 460 & $<3$ & - & Ausente & 3100 & - \\
\hline & $\mathbf{M}$ & $<3$ & $<3$ & - & Ausente & 7600 & - \\
\hline & $\mathbf{N}$ & $<3$ & $<3$ & - & Ausente & - & - \\
\hline & 0 & 1100 & $<3$ & - & Ausente & - & - \\
\hline \multirow{4}{*}{$\begin{array}{l}\text { Região } \\
\text { Periférica }\end{array}$} & $\mathbf{P}$ & $<3$ & $<3$ & - & Ausente & - & - \\
\hline & $\mathbf{Q}$ & 460 & $<3$ & - & Ausente & - & - \\
\hline & $\mathbf{R}$ & 20 & $<3$ & - & Ausente & 950 & - \\
\hline & $S$ & $\geq 1100$ & $<3$ & - & Ausente & 1550 & - \\
\hline RDC $n=12$ & & NE & $5 \times 10$ & NE & Ausente & NE & $5 \times 10^{2}$ \\
\hline
\end{tabular}

NMP/g: Número mais provável por grama; UFC/g: unidade formadora de colônia por grama; Termo.: Termotolerante; CN: Coagulase negativa; CP: Coagulase positiva. RDC: Resolução da Diretoria Colegiada. NE: Não estabelecido.

Após 30 dias, foram realizadas novas coletas nas mesmas sorveterias e os resultados foram parecidos. Das amostras pertencentes às sorveterias da região central da cidade, apenas uma não estava contaminada por coliformes totais e duas não estavam contaminadas por estafilococos, entretanto, foram 
encontradas espécies de $E$. coli e de Estafilococos coagulase positiva como demostrado na tabela 2. Já nas sorveterias do centro comercial, apesar da presença de Estafilococos coagulase positiva em quatro amostras, os valores estavam dentro dos limites estabelecidos. Assim como da primeira vez, nas sorveterias da região periférica da cidade, não foram observados microrganismos patogênicos estando todas as amostras dentro dos padrões de qualidade.

Tabela 2 - Qualidade microbiológica de sorvetes comercializados em sorveterias analisados 30 dias depois.

\begin{tabular}{|c|c|c|c|c|c|c|c|}
\hline \multirow{2}{*}{ Região } & \multirow{2}{*}{ Sorveteria } & \multicolumn{3}{|c|}{ Coliformes (NMP/g) } & \multirow{2}{*}{ Salmonella sp. } & \multicolumn{2}{|c|}{ Estafilococos (UFC/g) } \\
\hline & & Totais & Termo. & E. coli & & $\mathrm{CN}$ & $\mathbf{C P}$ \\
\hline \multirow{7}{*}{$\begin{array}{l}\text { Região } \\
\text { Central }\end{array}$} & A & 4 & $<3$ & - & Ausente & - & - \\
\hline & B & 460 & 4 & + & Ausente & 600 & $6 \times 10^{2}$ \\
\hline & C & 460 & $<3$ & - & Ausente & 6700 & - \\
\hline & D & $<3$ & $<3$ & - & Ausente & 100 & - \\
\hline & $\mathbf{E}$ & 9 & $<3$ & - & Ausente & 300 & $3 \times 10^{2}$ \\
\hline & $\mathbf{F}$ & 460 & $<3$ & - & Ausente & - & - \\
\hline & $\mathbf{G}$ & 240 & 23 & + & Ausente & 300 & $3 \times 10^{2}$ \\
\hline \multirow{7}{*}{$\begin{array}{c}\text { Centro } \\
\text { Comercial }\end{array}$} & $\mathbf{H}$ & 460 & $<3$ & - & Ausente & - & - \\
\hline & $\mathbf{I}$ & $<3$ & $<3$ & - & Ausente & - & - \\
\hline & J & 4 & $<3$ & - & Ausente & 100 & $1 \times 10^{2}$ \\
\hline & $\mathbf{L}$ & 240 & $<3$ & - & Ausente & 400 & $4 \times 10^{2}$ \\
\hline & M & $<3$ & $<3$ & - & Ausente & 700 & - \\
\hline & $\mathbf{N}$ & 9 & $<3$ & - & Ausente & 100 & $1 \times 10^{2}$ \\
\hline & 0 & 150 & $<3$ & - & Ausente & 500 & $5 \times 10^{2}$ \\
\hline \multirow{4}{*}{$\begin{array}{c}\text { Região } \\
\text { Periférica }\end{array}$} & $\mathbf{P}$ & $<3$ & $<3$ & - & Ausente & 5000 & - \\
\hline & $\mathbf{Q}$ & $\geq 1100$ & $<3$ & - & Ausente & - & - \\
\hline & $\mathbf{R}$ & 240 & $<3$ & - & Ausente & 2750 & - \\
\hline & $\mathrm{S}$ & $\geq 1100$ & $<3$ & - & Ausente & 1600 & - \\
\hline RDC no 12 & & NE & $5 \times 10$ & NE & Ausente & NE & $5 \times 10^{2}$ \\
\hline
\end{tabular}

NMP/g: Número mais provável por grama; UFC/g: unidade formadora de colônia por grama; Termo.: Termotolerante; CN: Coagulase negativa; CP: Coagulase positiva. RDC: Resolução da diretoria colegiada. NE: Não estabelecido.

De acordo com Silva e colaboradores (2017), microrganismos do grupo Coliformes são os principais colonizadores do trato gastrointestinal de seres humanos e são indicadores das condições higiênico-sanitárias de alimentos, indicando erros na manipulação do produto. Silveira e 
colaboradores (2009) ressaltam que quanto maior for o número de bactérias coliformes, maior será a precariedade do processamento da matéria prima, com isto, o produto terá uma deterioração mais rápida, sendo um risco para a saúde da população.

Das 18 amostras analisadas no primeiro dia, apenas duas sorveterias da região central apresentaram valores acima dos limites estabelecidos pela resolução vigente para coliformes termotolerantes em que foram identificadas cepas de $E$. coli estando em desacordo com a mesma resolução. $\mathrm{Na}$ segunda coleta, houve presença de coliformes termotolerantes com limites dentro do permitido, porém, mesmo em pouca contagem, também foi verificada a presença do patógeno $E$. coli.

Nos estudos de Oliveira e colaboradores (2012), ao analisarem sorvetes comercializados em supermercados da cidade de Maceió $\mathrm{AL}$, foram constados valores acima dos padrões microbiológicos de $5 \times 10 \mathrm{NMP} / \mathrm{g}$ de contagens de coliformes termotolerantes, estando em desacordo com a legislação vigente, indicando condições higiênicas e sanitárias inadequadas, podendo ocasionar as DTAs. Pazianotti e colaboradores (2010) encontraram coliformes termotolerantes em valores que ultrapassaram em $100 \%$ os padrões vigentes para sorvetes artesanais e industriais comercializados na região de Arapongas-PR, enquanto que Hamú e Cardoso (2018) encontraram coliformes termotolerantes em $40 \%$ das amostras de sorvetes comercializados em Goiânia - GO.

Neste estudo foi encontrada, em três sorveterias da região central, a espécie $E$. coli sendo uma dessas sorveterias reincidente. Para Souza e colaboradores (2015) a presença desse microrganismo denotando contato de material de origem fecal com sorvete, devido provavelmente à falha na higiene tanto de manipuladores quanto de consumidores. Escherichia coli é um dos principais microrganismos responsáveis pelas DTAs, gerando um número considerável de vítimas, principalmente paciente imunodeprimidos. Um dos meios de transmissão mais comum é o consumo de alimentos contaminados (BATISTA et al., 2014).

Em todas as amostras de sorvete analisadas no presente estudo, em nenhuma foi encontrada espécimes de Salmonella spp. atendendo, portanto, resolução vigente que exige como padrão a ausência desse microrganismo. Há outros estudos sobre avaliação microbiológica de sorvetes, no qual os resultados encontrados são parecidos com os deste trabalho como no estudo de Hamú e Cardoso (2018), com sorvetes comercializados em Goiânia - GO, Degenhardt e Boff (2016) que não 
observaram presença de Salmonella spp. em pesquisa realizada com 32 amostras de sorvetes comercializados no município de Ouro - SC, Dias e colaboradores (2016) que analisaram amostras de sorvetes do tipo italiano comercializados na cidade de Fortaleza - CE e também verificaram ausência de Salmonella spp. e nos estudos de Santos e Verona (2014) que em todas as amostras analisadas de sorvetes de sabor creme comercializados na cidade de Francisco Beltrão - PR, não foram encontradas este microrganismo.

Nos estudos de Souza e colaboradores (2015), entretanto, foi encontrada a espécie Salmonella spp em nove amostras de sorvetes comercializados na cidade de Sinop - MT. Oliveira e colaboradores observaram o crescimento de colônias em suas placas, e após ser feito o teste de identificação bioquímica, todas as suas amostras apresentaram resultados positivos, confirmando a presença de Salmonella spp. Nos estudos de Silveira e colaboradores (2009), das quatro amostras analisadas, três foram confirmadas com a presença de Salmonella spp. e Queiroz e colaboradores (2009) de quatro amostras analisadas de sorvetes industrializados e comercializados em Fortaleza - CE, em três foram encontraram espécies desse microrganismo.
De acordo com Oliveira e colaboradores (2013), os fatores de virulência da Salmonella spp. dependem do estado imunológico do hospedeiro, podendo ocasionar desde uma infecção gastrointestinal branda até uma infecção sistêmica. Depois de uma contaminação oral, o patógeno se prolifera no intestino delgado, invadindo a mucosa intestinal e destruindo a camada epitelial. Diversas subespécies patogênicas de Salmonella spp. podem causar de fortes cólicas, febre, e diarreia sanguinolenta em casos mais graves (TONG et al., 2015).

Com relação aos estafilococos, neste estudo $72,22 \%$ das amostras de sorvetes estavam contaminadas por este microrganismo sendo que desse total, $38,46 \%$ eram Estafilococos coagulase positiva. Das 18 amostras coletadas no primeiro dia, três deram positivos para Estafilococos coagulase positiva onde os valores ultrapassam abusivamente os padrões da resolução vigente. $\mathrm{Na}$ segunda coleta, em apenas uma amostra os valores ultrapassaram os limites.

Oliveira, Lyra, Esteves (2013) verificaram altas contagens de $S$. aureus em amostras coletadas em Maceió - AL. Hamú e Cardoso (2018) encontrou em $20 \%$ das amostras a presença de estafilococos coagulase positiva. Paiva e colaboradores (2016) verificaram a presença de Staphylococcus spp. fora dos padrões exigidos em sorvetes do tipo 
italiano comercializados em Pombal - PB.

Marinho e colaboradores (2017) encontraram em três amostras de sorvete comercializadas na cidade de São Luís $\mathrm{MA}$, números elevados de até $10^{5} \mathrm{UFC} / \mathrm{g}$ de sorvete. No estudo de Queiroz e colaboradores (2009), em todas as amostras analisadas houve presença desse microrganismo com valores que ultrapassam os limites da resolução vigente. Diferente desses estudos, as autoras Santos e Verona (2014) observaram que todas as análises de amostras de sorvetes apresentaram-se dentro do padrão exigido pela Legislação vigente para estafilococos coagulase positiva.

A espécie Staphylococcus aureus é um patógeno responsável por diversos casos de intoxicações devido a sua capacidade de produzir enterotoxinas termoestáveis, que podem permanecer no alimento mesmo após o cozimento, possibilitando, desta forma, a instalação de um quadro de intoxicação de origem alimentar (TONG et al., 2015) podem gerar vômitos, náuseas e prostração em pouco tempo após a ingestão do alimento (NUNES; MOTA; CALDAS, 2013). As fontes mais comuns de contaminação por este microrganismo são as fossas nasais, mãos e braços dos manipuladores de alimentos (FERREIRA; LIMA COELHO, 2014).
Os resultados obtidos deste estudo atestam má qualidade microbiológica dos sorvetes comercializados e indicam as condições higiênicas em que este produto foi produzido e/ou comercializado. De acordo com Weber e Carrijo (2018) o sorvete é um alimento que passa por um processo de pasteurização, que se realizado de forma correta, presume-se que tais microrganismos sejam destruídos. Uma hipótese que não pode ser descartada é a recontaminação do produto ocorrida durante as etapas do processamento subsequentes à pasteurização. Outra observação muito importante é de se atentar com as condições pós-processamento, sobretudo, a manutenção da temperatura de refrigeração indicada para a conservação do produto. Podemos ainda sugerir que as elevadas populações observadas neste estudo poder ter como causa uma possível deficiência nas condições higiênico-sanitárias dos estabelecimentos envolvidos na comercialização dos sorvetes, sugerindo, contato com conteúdo fecal, seja através de matériaprima ou de equipamentos mal higienizados, ou de manipulação higiênica inadequada.

Weber e Carrijo (2018) ainda chamam a atenção para a capacitação dos manipuladores para a adequada aplicação das boas práticas de produção e manipulação, adequada higienização 
dos equipamentos, controles de tempo e temperatura do produto, sendo estas condutas fundamentais para que as contagens das populações microbianas pesquisadas sejam menores.

\section{CONCLUSÃO}

Das amostras analisadas provenientes das 18 sorveterias pesquisadas, 4 foram reprovadas, sendo uma delas reincidente, por estarem com limites de contaminação microbiológica acima dos estabelecidos pela legislação. As amostras analisadas obtidas de sorveterias da região central foram as mais contaminadas neste estudo, onde foram encontradas a presença de $E$. coli e Estafilococos coagulase positiva. A presença desses microrganismos sugere falhas em algum momento do processamento, armazenamento ou da venda destes produtos tornando-os inadequados, podendo não só causar uma deterioração mais rápida do sorvete, mas também colocando em risco a saúde do consumidor.

\section{REFERÊNCIAS BIBLIOGRÁFICAS}

APHA (AMERICAN PUBLIC HEALTH ASSOCIATION). Committe on Microbiologycal Methods for Foods. Compendiun of Methods for the Microbiologycal Examination of Foods. Washington: APHA, 2015.
BATISTA, S. A.; BARROS, S. S. L.; SANTOS, V. S. E; SILVA, H. M. Escherichia coli 0157:H7 em leite produzido no Brasil. Revista brasileira de higiene e sanidade animal. v.8, n.2, p. 87-111, 2014

BRASIL. Ministério da Saúde. ANVISA Agência Nacional de Vigilância Sanitária. Regulamento técnico sobre padrões microbiológicos para alimentos RDC $\mathrm{n}^{\circ}$ 12. Diário Oficial da União; Poder Executivo, janeiro de 2001.

BRASIL. Ministério da Saúde. ANVISA Agência Nacional de Vigilância Sanitária. regulamento técnico de boas práticas de fabricação para estabelecimento industrializadores de gelados comestíveis e a lista de verificação das boas práticas de fabricação para estabelecimentos industrializadores de gelados comestíveis RDC nํ 267. Diário Oficial da União; Poder Executivo, janeiro de 2003.

DEGENHARDT, R. T.; BOFF, J. B. Qualidade Microbiológica de Sorvetes comercializados em Buffet e Tipo Expresso. In: Anais do XXII Seminário de Iniciação Científica. Universidade do Oeste de Santa Catarina. Joaçaba, Brasil. 2016.

DIAS, F. G. B.; PEREIRA, E. C.; FERREIRA, M. J. G.; ARAÚJO, D. C.; QUEIROZ, L. O.; FIGUEIREDO, E. A. T. Condições higiênico-sanitárias e pesquisa de Listeria monocytogenes em sorvetes do tipo italiano comercializados na cidade de Fortaleza. In: Anais do 25ํㅡㄹ Congresso Brasileiro de Ciência e Tecnologia de Alimentos. Sociedade Brasileira de Ciência e Tecnologia de Alimentos. Gramado, Brasil. 2016.

DIOGO, T. G.; AGUIAR, M. G.; TOLENTINO, C. M.; BUFFARA, D.; PILEGGI, M.; Avaliação microbiológica de sorvetes comercializados na cidade de Ponta Grossa - PR e da água usada na limpeza das colheres utilizadas para servilos. Biological and Health Sciences. v.8, n.1, p.23-32, 2002. 
FALCÃO, P. D.; FILHO, S. G.; NISHIDA, K. N.; BORGES, R. S. Exame microbiológico de sorvetes não pasteurizados. Saúde publica. n.17, p.28, 1983.

FERREIRA, H.; LIMA, H.; COELHO, T. Microrganismos indicadores em alimentos de origem animal. Programa de Pós-Graduação em Ciência Animal (PPGCA) da Universidade Federal Rural do Semi-Árido (UFERSA), 2014, 10 f.

HAMÚ, J. R. P. N.; CARDOSO, A. M. Avaliação microbiológica de sorvetes comercializados em Goiânia-GO. RBAC. v.50, n.4, p.351-357, 2018;

MARINHO, S. C.; FERREIRA, C. E. da S.; NUNES, G. S.; SILVA, C. N. C.; SILVA, G. M. Avaliação microbiológica do sorvete artesanal de coco comercializado na cidade de São Luís - MA. Higiene Alimentar, v.31, n.266/267, p.2741, 2017.

NUNES, M. M.; MOTA, A. L. A. A.; CALDAS, E. D. Investigation of food and water microbiological conditions and foodborne disease outbreaks in the Federal District, Brazil. Food Control. v.34, p.235-240, 2013.

OLIVEIRA, F. M.; Lyra, I. N.; Esteves, G. S. G. Avaliação microbiológica e físicoquímica de iogurtes de morango industrializados e comercializados no município de Linhares-ES. Revista Brasileira de Produtos Agroindustriais. v.15, n.2, p.147-155, 2013.

OLIVEIRA, S. E.; MARQUES, P. J. L.; SANTOS, S. R. E.; GALDINO, N. M. R. Pesquisa de coliformes totais e termotolerantes em águas minerais envasadas, comercializadas na cidade do Recife-PE. In: XIII Jornada de ensino, pesquisa e extensão. Recife. 2013.

OLIVEIRA, T. E.; BATISTA, S. J. P.; OLIVEIRA, G. E.; SILVA, F. T. I.; FROEHLICH, A. Avaliação microbiológica de sorvetes comercializados nos principais supermercados de Maceió - AL. In: Congresso Norte e Nordeste de pesquisa e inovação. Tocantins. 2012.

PAIVA, Y. F.; SILVA, E. V.; ARAÚJO, A. S.; AZEVEDO, P. T. M.; SOUSA, T. C. A. Condições Higiênico-Sanitárias de Sorvetes do Tipo Italiano (Soft), comercializados em Pombal, Paraíba. Revista Verde. v.11, n.3, p.75-79, 2016.

PAZIANOTTI, L.; BOSSO, A. A.; CARDOSO, S.; COSTA, M. R.; SIVIERI, K. Características Microbiológicas e Físico-Químicas de Sorvetes Artesanais e industriais comercializados na região de Arapongas-PR. Rev Inst Latic. v.65, n.377, p.15-20, 2010.

QUEIROZ, H. G. S.; SAMPAIO NETA, N. A.; PINTO, R. S.; RODRIGUES, M. C. P.; COSTA, J. M. C. Avaliação da qualidade físicoquímica e microbiológica de sorvetes do tipo tapioca. Revista Ciência Agronômica. v.40, n.1, p.60-65, 2009.

RHA (REVISTA HIGIENE ALIMENTAR). Consumo de sorvete aumentou no Brasil. Disponível em: https://higienealimentar.com.br/consumode-sorvete-aumentou-no-brasil/. Acesso em: 27 de setembro de 2021.

SANTOS, T. C.; VERONA, V. Avaliação microbiológica e química de sorvetes de sabor creme comercializados na cidade de Francisco Beltrão-PR. Monografia (Graduação de Tecnologia em Alimentos) Universidade Tecnológica Federal do Paraná; 2014.52p.

SILVA, N.; JUNQUEIRA, V. C. A., SILVEIRA, N. F. A., TANIWAKI, M. H., GOMES, R. A. R., OKAZAKI, M. M. Manual de Métodos de Análises Microbiológica de Alimentos e Água. $5^{\mathrm{a}}$ ed., São Paulo, 2017, 535 p.

SILVEIRA, G. H.; NETA, S. A. N.; PINTO, S. R.; RODRIGUES, P. C. M.; COSTA, C. M. J.; Avaliação da qualidade físicoquímica e microbiológica de sorvetes do 
tipo tapioca. Revista Ciência agronômica. v.40, n.1, p. 60-65, 2009.

SOUZA, J. M.; BRITO, N. J. N.; SANTOS, E. C. G.; SILVA, G. A. Análise microbiológica dos sorvetes self-service sabor chocolate da cidade de Sinop-MT. Demetra, v.10, n.4, p.857-66, 2015.

TONG, S. Y.; DAVIS, J. S.; EICHENBERGER, E.; HOLLAND. T. L.; FOWLER, V. G. Staphylococcus aureus infections: epidemiology, pathophysiology, clinical manifestations and management. Clinical Microbiology Reviews. v.28, n.3, p.603-61, 2015

WEBER, C.; CARRIJO, K. F. Análise microbiológica de sorvete expresso comercializados em Uberlândia, minas gerais, brasil. Enciclopédia biosfera, Centro Científico Conhecer - Goiânia, v.15, n.28, p.58, 2018. 